OPEN

SUBJECT AREAS:

SUB-WAVELENGTH

OPTICS

QUANTUM DOTS

NANOPHOTONICS AND

PLASMONICS

Received

19 June 2014

Accepted

11 August 2014

Published

24 September 2014

Correspondence and requests for materials should be addressed to

J.M.G. (igerton@

physics.utah.edu)

\section{Using a Sharp Metal Tip to Control the Polarization and Direction of Emission} from a Quantum Dot

\author{
Anil Ghimire, Eyal Shafran \& Jordan M. Gerton
}

Department of Physics and Astronomy, University of Utah, Salt Lake City, USA.

Optical antennas can be used to manipulate the direction and polarization of radiation from an emitter. Usually, these metallic nanostructures utilize localized plasmon resonances to generate highly directional and strongly polarized emission, which is determined predominantly by the antenna geometry alone, and is thus not easily tuned. Here we show experimentally that the emission polarization can be manipulated using a simple, nonresonant scanning probe consisting of the sharp metallic tip of an atomic force microscope; finite element simulations reveal that the emission simultaneously becomes highly directional. Together, the measurements and simulations demonstrate that interference between light emitted directly into the far field with that elastically scattered from the tip apex in the near field is responsible for this control over polarization and directionality. Due to the relatively weak emitter-tip coupling, the tip must be positioned very precisely near the emitter, but this weak coupling also leads to highly tunable emission properties with a similar degree of polarization and directionality compared to resonant antennas.

T he function of an optical antenna is to reduce the impedance mismatch between propagating (far-field) radiation modes and non-propagating (near-field) evanescent modes, which allows an emitter to absorb and/or emit light more efficiently ${ }^{1,2}$. A consequence of the coupling between an antenna and an emitter is that the radiation pattern and/or polarization can be strongly modified ${ }^{3-13}$. This phenomenon is fundamentally interesting, and forms a basis for developing single photon sources for quantum information applications ${ }^{14-16}$. Optimally, antennas would provide simultaneous control over the direction, phase, and polarization of individual photons emitted from a source, requiring a comprehensive understanding of the emitter-antenna coupling. Here, we demonstrate that the sharp, nonresonant tip of an atomic force microscope (AFM) can be used to simultaneously control the polarization and direction of emission from individual quantum emitters. In contrast to resonant antennas where the large local density of optical states (LDOS) leads to emission properties dominated by the antenna modes, the much smaller LDOS values for nonresonant tips leads to an emission pattern and polarization that depend very sensitively on the position of the tip and the orientation of the emitter. When separated by an intermediate distance, the emitter and tip contribute roughly equally to the emission properties, and we show that maximum control over the emission direction and polarization is achieved in this intermediate zone, with performance similar to resonant antennas but with much better tunability.

In one of the earliest demonstrations, Gersen et al. used the annular disk of an aperture-type near-field probe to control the emission pattern from single molecules ${ }^{3}$ and later this group used a similar setup with polarization analysis to demonstrate reversible polarization control ${ }^{7}$. Other work falls mostly into two general categories: nonscannable but highly directional antennas ${ }^{12}$ and scannable resonant antennas, which include both linear ${ }^{6,17}$ and spherical $^{5,18,19}$ geometries. In the first category, Curto et al. used a multi-element Yagi-Uda antenna to generate highly directional, strongly polarized emission from a single QD, but because that QD was painstakingly positioned lithographically, it was difficult to study the emission properties as a function of the QD position. In the second category, Taminiau et al. and Neumann et al. used scanning single-element dipole antennas to strongly modify the emission pattern, which was dominated by the resonant dipolar modes of the antenna, and was thus neither highly directional nor very tunable ${ }^{6,17}$. Kuhn et al. used a resonant gold nanosphere (50 nm diameter) attached to the end of a glass fiber to control the emission polarization and directionality as the sphere was scanned in the vicinity of isolated emitters ${ }^{5,18,19}$. Kuhn et al. also attempted to use nonresonant sharp tips, but no change in the emission pattern was detected ${ }^{18}$, most likely because without a resonance, these tips must be brought much closer to the emitter to control emission properties, which leads to strong quenching of the fluorescence (non-radiative energy transfer). This problem is exacerbated when the emission is imaged directly (as was done by 
Kuhn et al.), since the scarce photon signal is then spread over the face of the camera resulting in very low signal-to-noise ratios. Here, we employ a novel single-photon counting technique ${ }^{20}$ coupled with polarization anisotropy measurements and finite difference time domain (FDTD) simulations to show that despite strong quenching, sharp nonresonant tips can be used to generate highly directional and strongly polarized emission from isolated QDs.

\section{Results}

Polarization control. The fluorescence emission from isolated QDs is separated into two orthogonal polarization channels (Fig. 1) and the degree of polarization (DOP) is calculated as:

$$
\mathrm{DOP}=\frac{I_{x}-I_{y}}{I_{x}+I_{y}}
$$

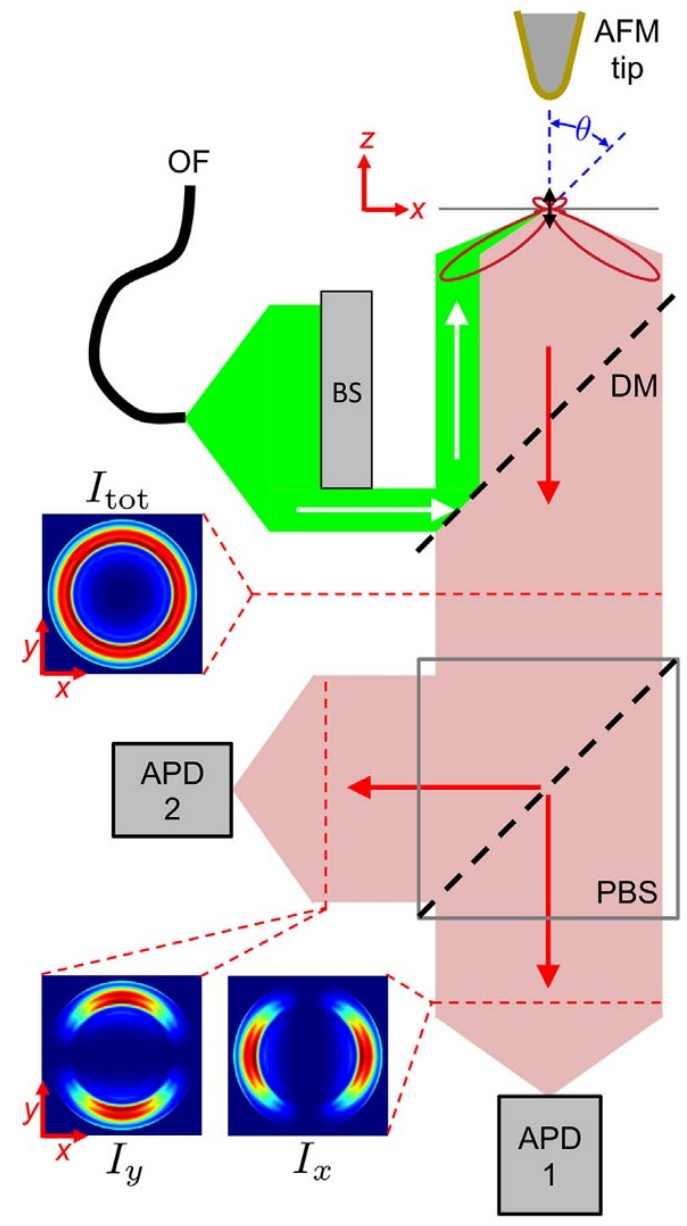

Figure 1 | Schematic of experimental setup. Laser light from an optical fiber (OF) is collimated, directed through a beam stop (BS), and then reflected from a dichroic mirror (DM). An oil-immersion microscope objective $(\mathrm{NA}=1.4)$ focuses the laser beam and collects emitted fluorescence. An AFM tip is positioned near an illuminated QD and the AFM tip is driven vertically in intermittent contact mode at $\sim 65 \mathrm{kHz}$ oscillation frequency. The collected emission passes through the DM and is incident on a polarization beam splitter (PBS). The $x$ - and $y$-polarization components are measured separately using two avalanche photodiodes (APD) operated in single photon counting mode. A simulated polar plot of emission intensity for a $z$-oriented dipole at the air/glass interface is shown at the sample plane to help illustrate the geometry. Simulated back focal images for the same $z$-dipole are shown just after the objective $\left(I_{t o t}\right)$ and in the two legs of the polarization analyzer $\left(I_{x}\right.$ and $\left.I_{y}\right)$.
The arrival time of each photon detected in both the $x$ - and $y$ polarization channels is correlated with the vertical $(z)$ and horizontal $(x, y)$ position of the AFM probe relative to the QD under study. This enables the total fluorescence signal $\left(I_{x}+I_{y}\right)$ and the DOP to be plotted in the three dimensional half space above the sample surface ${ }^{20}$ as described in more detail below in the Methods section. Figure 2 shows experimental measurements of the total fluorescence signal (panel A) and DOP (panel B) from a QD as a function of the position of a gold-coated tip within an $x z$-plane that cuts through the center of the QD. The total emission from the QD is strongly quenched in the near field of the tip mainly due to nonradiative energy transfer followed by thermal dissipation in the tip $^{21}$. Clearly, the DOP decreases for small tip distances directly above the QD, indicating the emission polarization has equal $x$ and $y$ components. In contrast, when the tip is on either side of the QD, the DOP value increases, indicating that the emission becomes strongly polarized along the $x$ direction. We have performed $\sim 40$ DOP measurements on $>25$ QDs and the data shown here is very representative of the observed trends even though each QD measured exhibits a different DOP value in absence of the tip due to orientational variations. For example, all QDs measured exhibit an increase in the DOP magnitude when the tip is on either side of the QD, and a decrease in magnitude when the tip is just above the QD (e.g., see Fig. 3 inset). These observations are consistent with previous work using different tip/antenna geometries ${ }^{7,11}$.

Comparing Figs. 2A and 2B reveals a clear difference in the length scales associated with changes in the total fluorescence signal and DOP as well as a difference in how they depend on the location of the tip apex. When the tip approaches the QD from directly above, fluorescence quenching initiates when the tip is $\sim 60 \mathrm{~nm}$ above the QD whereas the emission polarization is not affected until the tip approaches to within $\sim 10 \mathrm{~nm}$, as demonstrated by the red curves in Fig. 3. Furthermore, when the tip approaches the QD from the side,
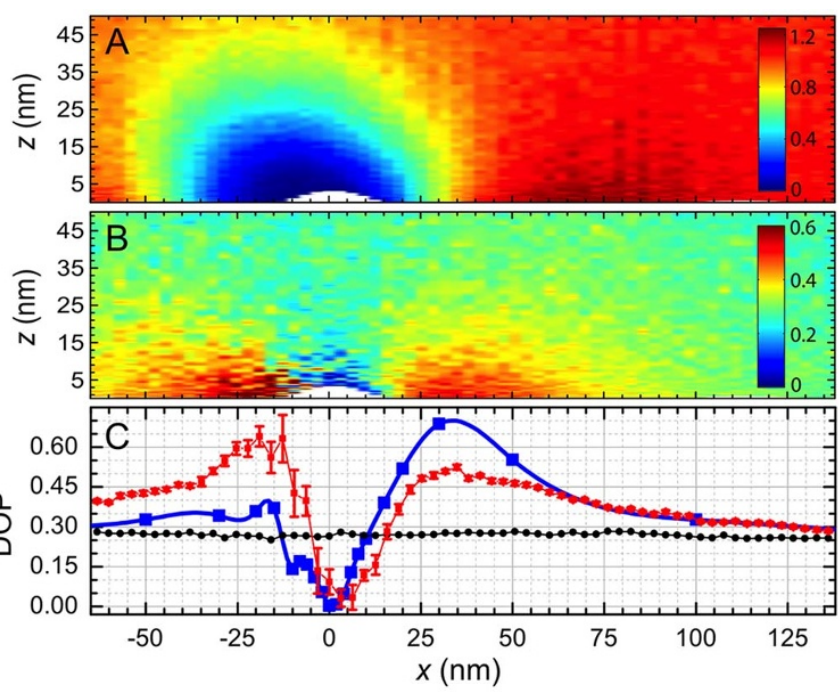

Figure $2 \mid$ High-resolution measurements of tip-induced polarization changes. (A) Fluorescence intensity from a QD $\left(I_{x}+I_{y}\right)$ as a function of tip position in an $x z$-plane through the center of the QD. The color bar gives the intensity normalized to the far-field intensity $(z>50 \mathrm{~nm})$ at each lateral $(x)$ position of the tip. (B) Corresponding DOP image calculated using Eq. 1. (C) Section plots from panel B for $z>50 \mathrm{~nm}$ (black symbols) and $z<2 \mathrm{~nm}$ (red symbols). Blue squares depict the simulated DOP for a gold tip (with $15 \mathrm{~nm}$ radius of curvature) maintained $2 \mathrm{~nm}$ above the air/ glass interface and a dipole emitter tilted by $\theta=45^{\circ}$ relative to the vertical in the $x z$-plane. The white space in panels A and B represent the AFM height trace and the blue line in panel $\mathrm{C}$ is a guide to the eye. 
the DOP initially increases reaching a peak value when the tip is 20$35 \mathrm{~nm}$ on either side of the QD, whereas the total fluorescence signal decreases fairly monotonically as the tip approaches. Interestingly, when a single carbon nanotube (CNT) is used as an AFM probe ${ }^{22-24}$, it does not affect the polarization at all even though the CNT strongly quenches the QD emission (black curves in Fig. 3).

When the tip is laterally displaced from the QD (along $x$ in Fig. 2C), we observe a substantial increase in the DOP indicating predominantly $x$-polarized signal photons. We have also acquired DOP data when the tip is scanned in a $y z$-plane (see supplemental information), and the resulting images exhibit similar features to those shown in Fig. $2 \mathrm{~B}$ and $\mathrm{C}$, but the DOP values are negative, indicating the signal photons are predominantly $y$-polarized. Thus, as the tip is scanned laterally in the vicinity of the QD, the far-field emission polarization can be controlled such that it aligns with the axis separating the QD and tip apex.

Directional control. To investigate directional control of the emission pattern, we used rigorous finite difference time domain (FDTD) simulations (Lumerical, Inc.) to generate polarization and directional maps of the collected far-field emission. Although it would be ideal to measure the emission pattern directly using, e.g., back focal imaging as the tip is scanned over an isolated QD, such a direct measurement is difficult to achieve in combination with the high-precision photon counting technique employed here. The simulation is used to generate the emission pattern (back focal images) and the emission directionality $(D)$ is quantified as the ratio of emission intensity at two diametrically opposite points in the back focal images, either along the $x$-axis $\left(D_{x}\right)$ or the along the $y$ axis $\left(D_{y}\right)$ :

$$
D_{x}=10 \log _{10}\left(\frac{I_{+x}}{I_{-x}}\right)
$$

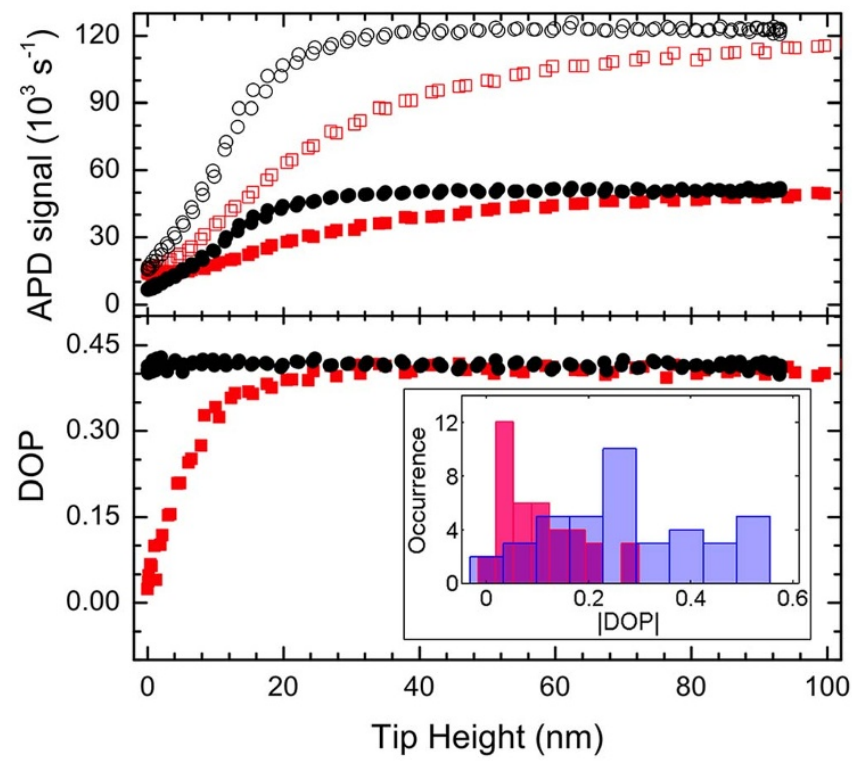

Figure 3 | Vertical DOP approach-curve measurements for a carbon nanotube tip (black circles) and a gold tip (red squares). These measurements were performed on two different QDs that have the same asymptotic DOP value. Upper: The open (closed) symbols correspond to photon count rates on the $x(y)$ APD channel as the tip is lowered onto a QD from above. Lower: DOP values computed from the data in upper panel. Inset: histogram of $\sim 40$ DOP measurements when a gold tip is far above the QD (blue bars) and within $2 \mathrm{~nm}$ of the QD (red bars).

$$
D_{y}=10 \log _{10}\left(\frac{I_{+y}}{I_{-y}}\right)
$$

$D_{x}$ and $D_{y}$ are measured in decibels and also referred to as front-toback ratios. The simulated and measured DOP curves in Fig. 2C qualitatively agree with each other, which validates the simulations of emission patterns and directionality. The quantitative difference in magnitude and asymmetry between the simulated and measured DOP values are due to the simplified model for the QD (linear instead of $2 \mathrm{D}$ degenerate dipole $\mathrm{e}^{25,26}$ ) and also our simplified model for the tip as a vertically-oriented cone instead of a tilted and asymmetric pyramid; nonetheless, the strong qualitative agreement yields critical insights into the pertinent physics. Details of the simulations and post processing are described below in the Methods section.

Figure 4 shows a sample of simulated back focal images for different lateral positions of the gold tip for the conditions described above. The vertical tilt angle, $\theta$, of the dipole is adjusted to $45^{\circ}$ to give an asymptotic DOP value matching the particular QD under study (Fig. 2C). The lower panel in Fig. 4 quantifies the directionality of the emission for tip positions at the specified points along the $x$ - (blue squares) and $y$ - (green squares) axes, and the labeled points $\mathrm{A}-\mathrm{E}$ correspond to five of the back focal image panels; the sixth panel labeled " $\infty$ " shows the results of a simulation with no tip. The simulations reveal that the emission pattern can be strongly altered by the presence of the tip: photons are usually "steered" toward the tip ${ }^{19,27}$ with some limited regions (e.g., point A) where the photons are steered away from the tip. For the specific emitter orientation considered here $\left(\theta=45^{\circ}\right)$, there is an optimal tip-emitter lateral sepa-
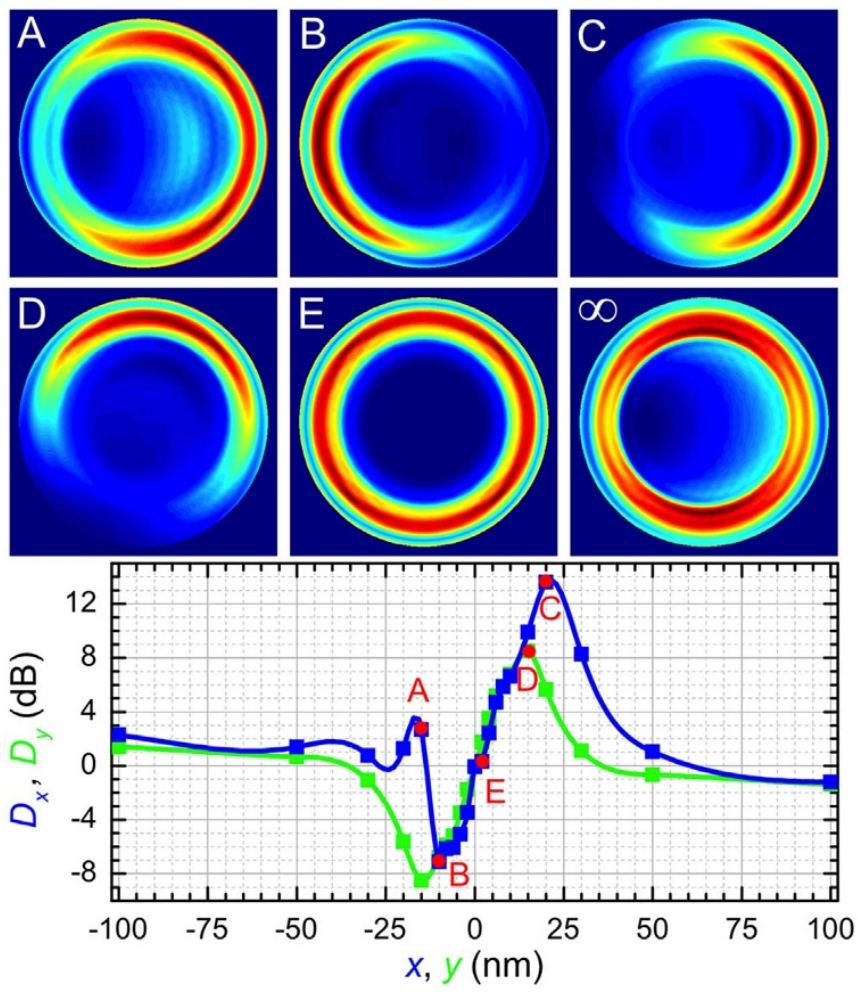

Figure $4 \mid$ Directional control of emission from a dipole using a gold tip. In these simulations, the dipole has a tilt angle $\theta=45^{\circ}$ and is located at $(x$, $y, z)=(0,0,2) \mathrm{nm}$. The coordinates of the tip apex in nm are $(x, y, z)=$ $(-15,0,2),(-10,0,2),(20,0,2),(0,15,2)$, and $(0,0,4)$ for points (A-E), respectively. The lower panel shows the directionality as the tip is positioned along the $x$ and $y$ axes at the specified points. In absence of the tip (panel marked “ $\infty$ "), the unperturbed directionalities are $D_{x}=0.4$ and $D_{y}=0$. The green and blue curves in the lower panel are guides to the eye. 


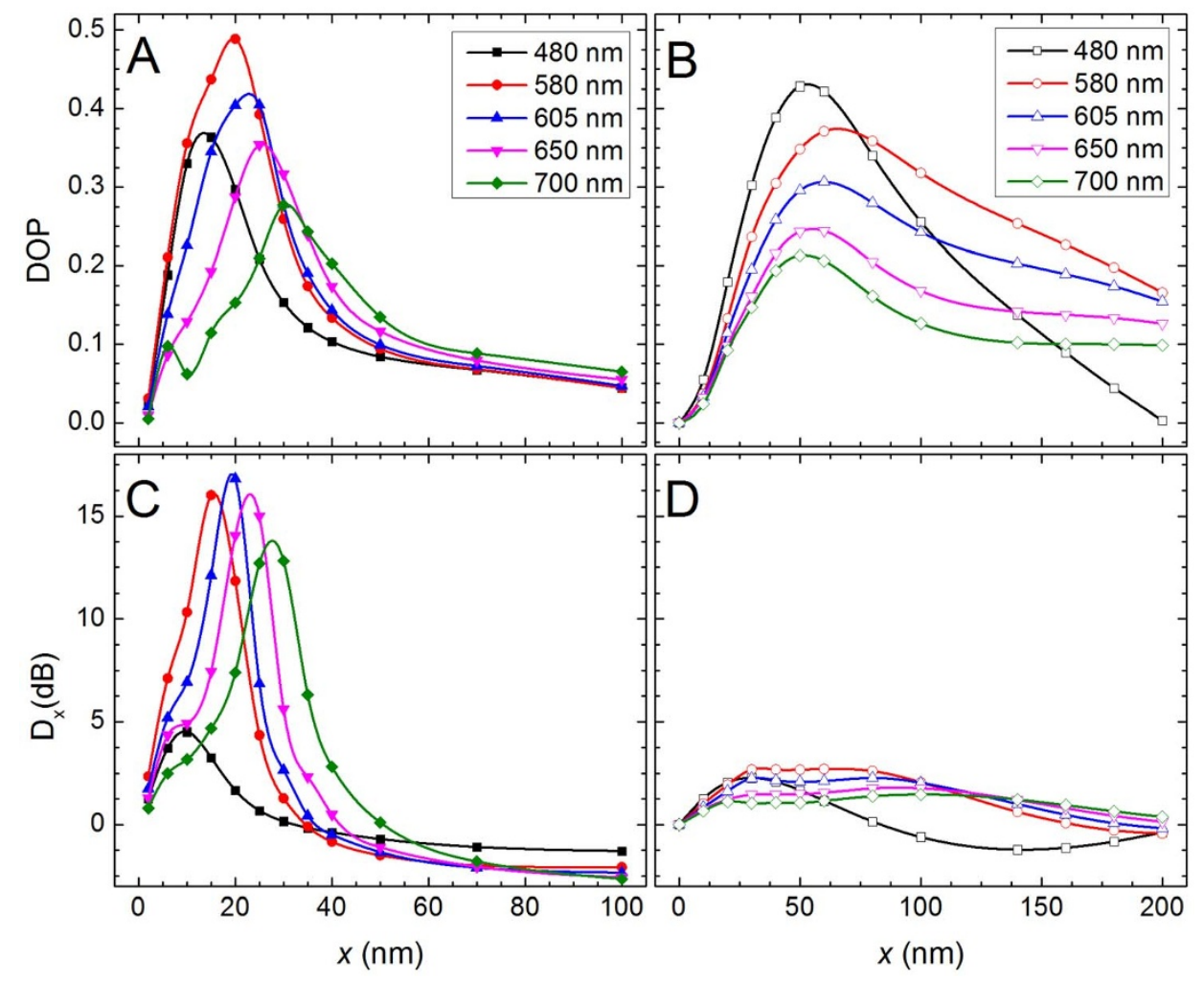

Figure $5 \mid$ DOP and directionality $\left(D_{x}\right)$ curves for a vertical dipole emitter with five different vacuum emission wavelengths. For all graphs, the black, red, blue, magenta, and green curves represent emission wavelengths of 480, 580, 605, 650 and $700 \mathrm{~nm}$, respectively. Panels (A) and (C) correspond to a sharp gold tip with $15 \mathrm{~nm}$ radius of curvature and panels (B) and (D) correspond to a gold nanosphere with radius $50 \mathrm{~nm}$. The horizontal axis corresponds to the lateral displacement of the tip/sphere apex relative to the dipole position (note the different scales between the right and left panels). The curves are guides for the eye.

ration that produces highly directional and strongly polarized emission, as shown in Figs. 2 and 4. Due to the asymmetry in the axial $(z)$ component of the field near a tilted dipole (see Supplementary Information), the tip must get closer to the emitter on one side ( $x$ $=-10 \mathrm{~nm})$ compared to the other $(x=+20 \mathrm{~nm})$ to achieve the conditions for optimal control over the emission direction and polarization.

Spectral response of a sharp tip compared to a nanosphere. Figure 5 shows the DOP (A and B) and directionality (C and D) for a sharp gold tip with $15 \mathrm{~nm}$ radius of curvature and a gold nanosphere with $50 \mathrm{~nm}$ radius for different lateral positions of the tip/sphere relative to a vertically oriented dipole emitter. The black, red, blue, magenta, and green curves represent emission wavelengths of 480, 580, 605, 650 and $700 \mathrm{~nm}$ wavelength respectively. Compared with the nanosphere, the AFM tip yields much better directional control and similar polarization control over a broad wavelength range. The AFM tip also yields much better control over both polarization and direction compared with a smaller nanosphere (15 $\mathrm{nm}$ radius), as shown in the Supplementary Information. The superior spectral response of the semi-infinite tip geometry is due, in part, to a lack of localized surface plasmon resonances, and in part to the strong vertical polarizability of the tip.

In contrast, the nanosphere has an isotropic polarizability, which prevents the formation of a strong linear dipole moment in the sphere, particularly since the near field of the emitter decays very rapidly in space, $\propto 1 / r^{3}$, leading to large field inhomogeneities within the sphere ${ }^{29}$. Furthermore, the coupling between an emitter and nanosphere is strongly modulated by plasmon resonances in the sphere, which have well-defined oscillation modes that generally do not lead to highly directional emission. The improved performance of the tip compared to the nanosphere requires better positional accuracy since the spatial dependance (Fig. 5) shows sharper peaks that occur at smaller tip-displacement values, $x$.

\section{Discussion}

As the tip approaches the QD in Figs. 2 and 3, the difference in characteristic length scale associated with the total fluorescence signal $\left(I_{x}+I_{y}\right)$ and DOP implies that there are two different physical mechanisms responsible for quenching and polarization/directional control. Indeed previous work has described two distinct emitter-tip coupling mechanisms: a short-range FRET-like dipole-dipole coupling and a longer-range effect associated with the generation of surface plasmon polaritons (SPPs) that propagate up the tip sidewalls ${ }^{28}$. We attribute the long-range quenching in our measurements (Figs. 2 and 3) mainly to the generation of propagating SPPs on the tip, while the short-range polarization/directional control is attributed to the dipole-dipole coupling mechanism. The "tip" dipole in this dipoledipole mechanism can be considered as an image of the source (emitter) dipole at the tip apex constrained by the dominant vertical polarizability of the tip. The magnitude of the tip dipole depends on the local strength and direction of the emitter's near-field, both of which fluctuate sharply near the emitter (see Supplementary Information).

We find that control over emission polarization and directionality is optimized when the source (emitter) and image (tip) dipoles have equal magnitude, are parallel to each other (as with the Yagi-Uda antenna), and are nearly $180^{\circ}$ out of phase with each other (Fig. 6). Nanospheres used as antennas lack a well-defined linear dipole moment, particularly when driven by the local source field of a nearby emitter, so control over directionality and polarization is sub-optimal (Fig. 5). On the other hand, a vertically oriented res- 


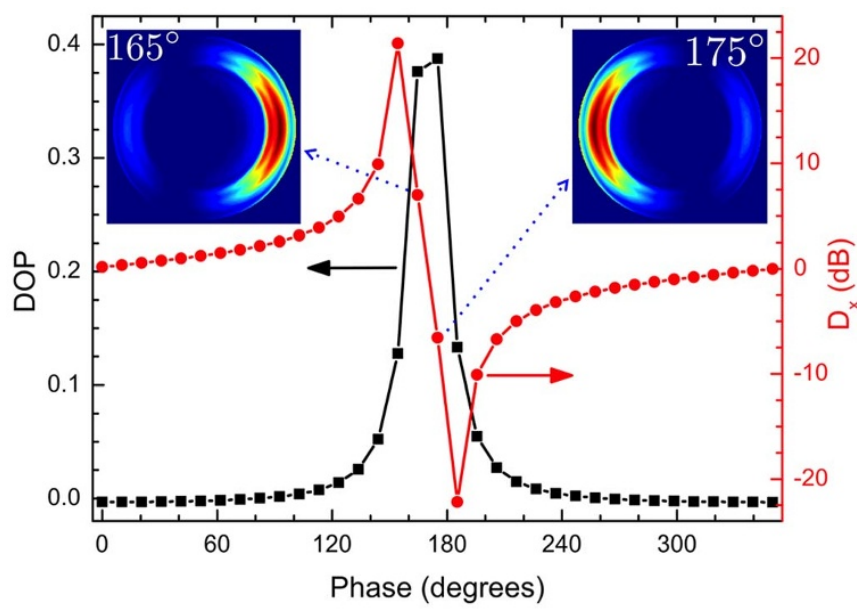

Figure 6 Simulated DOP and directionality for two identical vertical dipoles at $x=0$ and $x=20 \mathrm{~nm}$. Both dipoles are $2 \mathrm{~nm}$ above the air/glass interface. The phase of the dipole at $x=20 \mathrm{~nm}$ is varied with respect to the other. The insets show simulated back focal images for phase angles of $165^{\circ}$ and $175^{\circ}$.

onant dipole antenna has the requisite linear dipole moment, but due to strong plasmon resonances, it must be kept further from the emitter to achieve a similar dipole magnitude; this leads to a suboptimal phase relationship between the source and tip dipoles, resulting in less directional emission ${ }^{6,11}$. The sharp metallic tips used here posses a strong vertical dipole moment, and since they are nonresonant, they must be brought closer to an emitter to achieve a similar dipole magnitude; this leads to near optimal phase and magnitude relationships between the source and tip dipoles and strong control over emission polarization and directionality.

This dipole-dipole model suggests that the far-field radiation can be understood as the superposition of two source fields, one from the emitter and one scattered from the tip ${ }^{19,29,30}$. This idea is verified using a single-wall carbon nanotube (CNT) attached to an AFM probe since CNTs are strong fluorescence quenchers, but not strong light scatterers ${ }^{21}$. As Fig. 3 demonstrates, the CNT does not alter the emission polarization, nor presumably the directionality, when it approaches a QD. In contrast, the gold-coated tips used here are strong scatterers and although they strongly quench fluorescence, they also can be used to efficiently control the polarization and directionality of emission. As discussed above, the polarizability of an elongated tip is predominantly along its vertical axis ${ }^{29,31}$, leading to a dipole moment that by itself would produce radially polarized emission at the back focal plane (Fig. 1) and a DOP value near zero. Given this, it is evident from Figs. 2 and 3 that when a gold tip is lowered onto the QD from directly above, the collected signal is predominantly composed of photons scattered from the tip, while when the tip is displaced laterally by more than $\sim 5 \mathrm{~nm}$, the collected signal is the superposition of photons scattered from the tip and those emitted directly by the source.

In conclusion, we have demonstrated that the emission polarization and direction can be manipulated using a simple, nonresonant AFM probe. The mechanism responsible for this control is the interference between light emitted directly into the far field with that elastically scattered from the tip apex. Furthermore, due to the relatively weak emitter-tip coupling, the tip must be precisely positioned very close to the emitter, but this weak coupling also leads to highly tunable emission properties. Overall, the semi-infinite geometry of the tip yields superior polarization and directional control compared to either a metallic nanosphere or a resonant dipole antenna, and performs similarly to lithographically fabricated, highly-directional antennas. The ability to control the polarization together with the direction of emission could have important implications for the development of integrated nanophotonic devices and single photon sources.

\section{Methods}

Experimental setup and measurement. A schematic of the experimental setup is shown in Fig. 1. The overall design has a commercial AFM (Asylum Research MFP 3D) sitting atop a homebuilt inverted confocal optical microscope. The AFM tips used in this work are commercially available from BudgetSensors (part\# multi75B-G) and have a specified radius of curvature at the tip apex of $<25 \mathrm{~nm}$ (see Supplementary Information). A laser beam with wavelength $543 \mathrm{~nm}$ is incident on a beam stop (BS) that passes only supercritical rays through a high numerical aperture $(\mathrm{NA}=1.4)$ objective lens creating a focused, vertically polarized evanescent field at the sample plane. The AFM tip is aligned into the center of the focal spot and a single CdSe/ZnS QD ( $\lambda_{e m} \sim 605 \mathrm{~nm}$ from Invitrogen) is then positioned near the tip. The emitted fluorescence is collected by the same objective lens, and a polarization beam splitter (PBS) separates the emission into two orthogonal channels, each of which is monitored by an avalanche photodiode (APD). The detected photons are timestamped, and the arrival times are subsequently correlated with the lateral position of the tip and its height above the sample surface. Since the AFM tip oscillates vertically and the sample is scanned laterally, the photon count rate for each channel is measured as a function of the tip position in the three-dimensional half-space above the sample plane ${ }^{20}$. Separating the collected fluorescence into two orthogonal polarization channels allows the emission polarization to be monitored continuously, photon by photon, as the relative position between the tip and QD is controlled with $\sim 1 \mathrm{~nm}$ precision. To quantify the anisotropy between the two channels, we measured the degree of polarization (DOP) defined by Eqn. 1. The value of the DOP lies between -1 and +1 , with the extremes signifying linearly-polarized emission perfectly aligned with one channel or the other. The 2D measurements shown in Figs. 2 is produced by normalizing the count rate in each polarization channel to the average photon count rate for $z>50 \mathrm{~nm}$ for each lateral position of the tip after carefully subtracting the background count rate.

Simulation and emission pattern analysis. We used the FDTD simulation package from Lumerical Solutions Inc. In the simulations, a linear Hertzian dipole, with oscillation frequency corresponding to a free-space wavelength of $605 \mathrm{~nm}$, was situated $2 \mathrm{~nm}$ above an air/glass interface. A solid cylindrical gold cone with a $15 \mathrm{~nm}$ radius of curvature and full open-angle of $15^{\circ}$ was used to represent the AFM tip. The simulation domain was enclosed by a perfectly matched layer (PML) boundary to prevent reflections and backscattering from the domain edges. The total length of the cone is $1500 \mathrm{~nm}$ and the cone length within the simulation box is $450 \mathrm{~nm}$. The meshing size used in the simulation is $2 \mathrm{~nm}$. A near field monitor (NFM) was placed $50 \mathrm{~nm}$ below the interface within the glass to capture the field components and the near-fields are used to project the field components into the far-field regime giving the polarization and directional maps of the emission. The calculated far-field distribution is projected onto the $x-y$ plane perpendicular to the optical axis via a coordinate transformation to compute the field components $E_{x}$ and $E_{y}$ at the back focal plane of the microscope objective, and DOP values are then extracted for comparison with experimental measurements. More details regarding the simulations and post processing can be found in the supplementary information.

Once the simulated emission patterns (back focal images) are generated, the emission directionality $(D)$ is extracted using Eqn. 2. To compute $I_{+x}$ (or $I_{-x}$ ) we summed the simulated back-focal intensities within an angular window defined by an azimuthal range $-2^{\circ}<\phi_{+x}<2^{\circ}\left(178^{\circ}<\phi_{-x}<182^{\circ}\right)$ and a polar range $38^{\circ}<\theta<$ $68^{\circ}$; more than $80 \%$ of the total emission falls within this range of polar angles. $I_{+y}$ and $I_{-y}$ are computed in a similar fashion with the azimuthal ranges rotated by $90^{\circ}$. This procedure for extracting emission patterns from polarization anisotropy measurements using FDTD simulations is similar to that employed by Taminiau et al. ${ }^{6}$.

1. Schuck, P. J., Fromm, D. P., Sundaramurthy, A., Kino, G. S. \& Moerner, W. E. Improving the mismatch between light and nanoscale objects with gold bowtie nanoantennas. Phys. Rev. Lett. 94, 017402 (2005).

2. Mühlschlegel, P., Eisler, H.-J., Martin, O. J. F., Hecht, B. \& Pohl, D. Resonant optical antennas. Science 308, 1607-1609 (2005).

3. Gersen, H. et al. Influencing the angular emission of a single molecule. Phys. Rev. Lett. 85, 5312-5315 (2000).

4. Li, J., Salandrino, A. \& Engheta, N. Shaping light beams in the nanometer scale: A Yagi-Uda nanoantenna in the optical domain. Phys. Rev. B 76, 245403 (2007).

5. Kühn, S., Håkanson, U., Rogobete, L. \& Sandoghdar, V. Enhancement of singlemolecule fluorescence using a gold nanoparticle as an optical nanoantenna. Phys. Rev. Lett. 97, 017402 (2006)

6. Taminiau, T. H., Stefani, F. D., Segerink, F. B. \& Van Hulst, N. F. Optical antennas direct single-molecule emission. Nat. Photon. 2, 234-237 (2008).

7. Moerland, R. J., Taminiau, T. H., Novotny, L., van Hulst, N. F. \& Kuipers, L. Reversible polarization control of single photon emission. Nano Lett. 8, 606-610 (2008).

8. Biagioni, P. et al. Near-field polarization shaping by a near-resonant plasmonic cross antenna. Phys. Rev. B 80, 153409 (2009).

9. Li, Z., Shegai, T., Haran, G. \& Xu, H. Multiple-particle nanoantennas for enormous enhancement and polarization control of light emission. ACS Nano 3 , 637-642 (2009) 
10. Taminiau, T. H., Stefani, F. D. \& van Hulst, N. F. Enhanced directional excitation and emission of single emitters by a nano-optical Yagi-Uda antenna. Opt. Express 16, 10858-10866 (2008).

11. Taminiau, T. H., Stefani, F. D. \& van Hulst, N. F. Single emitters coupled to plasmonic nano-antennas: angular emission and collection efficiency. New J. Phys. 10, 105005 (2008).

12. Curto, A. G. et al. Unidirectional emission of a quantum dot coupled to a nanoantenna. Science 329, 930-933 (2010)

13. Kosako, T., Kadoya, Y. \& Hofman, H. F. Directional control of light by a nanooptical Yagi-Uda antenna. Nat. Photon. 4, 312-315 (2010).

14. Moerner, W. E. Single-photon sources based on single molecules in solids. New J. Phys. 6, 88 (2004).

15. Unitt, D. C. et al. Quantum dots as single-photon sources for quantum information processing. J. Opt. B: Quantum Semiclassical Opt. 7, 129 (2005).

16. Lounis, B. \& Orrit, M. Single-photon sources. Rep. Prog. Phys. 68, 1129 (2005)

17. Neumann, L., van t Oever, J. \& van Hulst, N. F. A resonant scanning dipoleantenna probe for enhanced nanoscale imaging. Nano Lett. 13, 5070-5074 (2013).

18. Kühn, S. \& Sandoghdar, V. Modification of single molecule fluorescence by a scanning probe. Appl. Phys. B 84, 211-217 (2006).

19. Kühn, S., Mori, G., Agio, M. \& Sandoghdar, V. Modification of single molecule fluorescence close to a nanostructure: radiation pattern, spontaneous emission and quenching. Mol. Phys. 106, 893-908 (2008).

20. Mangum, B. D., Shafran, E., Mu, C. \& Gerton, J. M. Three-dimensional mapping of near-field interactions via single-photon tomography. Nano Lett. 9, 3440-3446 (2009).

21. Shafran, E., Mangum, B. D. \& Gerton, J. M. Using the near-field coupling of a sharp tip to tune fluorescence-emission fluctuations during quantum-dot blinking. Phys. Rev. Lett. 107, 037403 (2011).

22. Hafner, J. H., Cheung, C.-L., Oosterkamp, T. H. \& Lieber, C. M. High-yield assembly of individual single-walled carbon nanotube tips for scanning probe microscopies. J. Phys. Chem. B 105, 743-746 (2001).

23. Mu, C., Mangum, B., Xie, C. \& Gerton, J. M. Nanoscale fluorescence microscopy using carbon nanotubes. IEEE J. Sel. Top. Quantum Electron. 14, 206-216 (2008).

24. Shafran, E., Mangum, B. D. \& Gerton, J. M. Energy transfer from an individual quantum dot to a carbon nanotube. Nano Lett. 10,4049-4054 (2010).

25. Empedocles, S., Neuhauser, R. \& Bawendi, M. Three-dimensional orientation measurements of symmetric single chromophores using polarization microscopy. Nature 399, 126-130 (1999).

26. Koberling, F. et al. Fluorescence anisotropy and crystal structure of individual semiconductor nanocrystals. J. Phys. Chem. B 107, 7463-7471 (2003).
27. Gersen, H. et al. Near-field effects in single molecule emission. J. Microsc. 202, 374-378 (2001).

28. Issa, N. A. \& Guckenberger, R. Fluorescence near metal tips: The roles of energy transfer and surface plasmon polaritons. Opt. Express 15, 12131-12144 (2007).

29. Novotny, L. \& Hecht, B. Principles of Nano-Optics (Cambridge University Press, 2006).

30. Bohren, C. \& Huffman, D. Absorption and Scattering of Light by Small Particles (Wiley, 1998).

31. Böhmler, M. et al. Enhancing and redirecting carbon nanotube photoluminescence by an optical antenna. Opt. Express 18, 16443-16451 (2010).

\section{Acknowledgments}

This work was supported by the National Science Foundation through a CAREER grant (DBI-0845193), a SCIALOG award from the Research Corporation for Science Advancement, and a seed grant from the University of Utah MRSEC program (DMR-1121252).

\section{Author contributions}

A.G. acquired all data for this work and performed all the analysis in consultation with E.S. and J.G.

\section{Additional information}

Supplementary information accompanies this paper at http://www.nature.com/ scientificreports

Competing financial interests: The authors declare no competing financial interests.

How to cite this article: Ghimire, A., Shafran, E. \& Gerton, J.M. Using a Sharp Metal Tip to Control the Polarization and Direction of Emission from a Quantum Dot. Sci. Rep. 4, 6456; DOI:10.1038/srep06456 (2014)

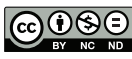

This work is licensed under a Creative Commons Attribution-NonCommercialNoDerivs 4.0 International License. The images or other third party material in this article are included in the article's Creative Commons license, unless indicated otherwise in the credit line; if the material is not included under the Creative Commons license, users will need to obtain permission from the license holder in order to reproduce the material. To view a copy of this license, visit http:// creativecommons.org/licenses/by-nc-nd/4.0/ 